\title{
Evaluation environnementale et sanitaire des aires d'abattage à l'heure de Covid-19 situées dans la commune de Matete ville-province de Kinshasa (RD Congo)
}

Umba M. Joachim ${ }^{1}, K_{\text {Kumpel M. Placide }}{ }^{2}$, Maleke M. Jean-Pierre ${ }^{3}$ et Metena M. Marlène ${ }^{1}$

${ }^{1}$ Université Pédagogique Nationale (UPN) B.P. 8855 Kinshasa-Ngaliema

${ }^{2}$ Laboratoire de Recherches des Sciences Humaines et des Sciences de l'Environnement, Centre de Recherche en sciences Humaines (CRESH) RDC.

${ }^{3}$ Institut Supérieur de Développement Rural (ISDR) de Mbeo/ Province du Kwilu

Email correspondant :joachimumba@yahoo.fr

Mots-clés : Evaluation sanitaire, environnement, aire d'abattage, Matete.

Keywords: Health assessment, environment, slaughter area, Matete.

Publication date 30/11/2020, http://m.elewa.org/Journals/about-japs /

\section{$1 \quad$ RÉSUMÉ}

Dans un monde de plus en plus interconnecté, la prévention contre les risques de maladies liées à la nourriture fait débat. Le contrôle de la qualité des aliments devient un problème de santé publique. Etant d'origine animale, Covid-19 qui, à ce titre est une zoonose émergente a suscité un grand intérêt scientifique. La question fondamentale qui se pose est de savoir si dans certains pays en développement la filière qui va du fermier éleveur, des abattoirs ainsi qu'à la chaine de distribution fait l'objet d'un marquage strict. Quelles pourraient-être les règles d'hygiène dans les abattoirs de certains pays telle que la RD Congo ?

Cet article propose d'évaluer, de faire un état de lieux des aires d'abattage de la Commune de Matete et faire vulgariser les règles de la sécurité sanitaire car la viande abattue dans ces tueries est exposée aux micro-organismes. Ils se dégagent que les tueries de la Commune de Matete sont caractérisées par des défaillances majeures concernant principalement le contrôle veterinaire et médical ,l'hygiène (du personnel, du lieu d'abattage et du matériel utilisé), la lutte contre les nuisibles et l'absence de système de collecte de sang et de traitement préalable des eaux usées, le rejet direct de ces effluents, en particulier dans le milieu hydrique, nuit gravement à la qualité de l'environnement.

\section{ABSTRACT}

In an increasingly interconnected world, prevention against the risks of food-related diseases is the subject of debate. Food quality control is becoming a public health issue. Being of animal origin, Covid-19 which, as such is an emerging zoonosis, has aroused great scientific interest.

The fundamental question that arises is to know whether in certain developing countries the chain which goes from the farmer-breeder, to the slaughterhouses as well as to the distribution chain is subject to strict marking. What could be the hygiene rules in slaughterhouses in some countries such as DR Congo?

this proposes to assess, make an inventory of the slaughter areas of the Municipality of Matete and popularize the rules of health security because the meat slaughtered in here is exposed 
to microorganisms. .They emerge that the slaughtering in the Municipality of Matete is characterized by major failures concerning mainly veterinary and medical control, hygiene (of the personnel, the place of slaughter and the equipment used), the fight against pests, the absence of blood collection system and pre-treatment of wastewater, the direct discharge of these effluents into the water environment, thus seriously affecting the environment.

\section{CONTEXTE ET OBJECTIF DE L'ETUDE}

Depuis décembre 2019, l'humanité fait face une maladie, la pandémie de COVID-19 qui a commencé en Chine et atteint la plupart des pays du monde. Etant d'origine animale, la COVID19 qui, à ce titre, est une zoonose émergente a suscité un grand intérêt scientifique. Dès le départ, l'origine de cette maladie avait été attribuée aux chauves-souris avec le pangolin comme hôte amplificateur. Qu'est-ce qu'un coronavirus? Les coronavirus forment une vaste famille de virus qui peuvent être pathogènes chez l'homme et chez l'animal, une zoonose Rubal Singla et al.,2020. La question qui se pose est de savoir quels enjeux et impacts que toutes ces maladies émergentes ont sur notre société. Cet article a pour objectif de savoir si dans quelles conditions fonctionnent ces tueries dans les Communes de Matete? Quelles sont les conséquences sanitaires environnementales liées au non-respect des principes sanitaires. Et observer si les étapes ante-mortem et postmortem étaient respectées et analyser si le service vétérinaire de l'Etat fait l'expertise ou l'inspection des viandes pour garantir les consommateurs. L'état et la bonne tenue des tueries sont souvent un indicateur de sécurité sanitaire. Par ailleurs, s'ils sont mal tenus, ces lieux sont susceptibles de contaminer la viande qui était de bonne qualité à l'origine et faciliter

\section{DÉMARCHE MÉTHODOLOGIQUE}

En République Démocratique du Congo, la lutte contre Covid-19 a démarré en mars 2020 après qu'un premier cas ait été constaté auprès d'une personne qui revenait de l'Europe. Depuis cette période, des mesures ont été prises en République Démocratique du Congo pour lutter contre l'épidémie

3.1 Le recueil de l'information: La collecte des données a privilégié le recueil d'informations auprès de différentes personnes aussi l'émergence de certaines zoonoses telle la COVID-19. La contamination des aliments par les micro-organismes se trouve facilitée par les conditions de l'environnement dans lequel ils sont vendus dans divers marchés de Kinshasa ou produits dans certains milieux. Les mesures d'hygiène ne sont pas respectées et la vente s'effectue dans un environnement insalubre, propice à la prolifération des microbes. Par ailleurs, les micro-organismes sont présents dans l'environnement naturel de l'homme (eau, sol, surfaces diverses), sur l'homme lui-même et sur les êtres vivants, plantes, animaux, d'où ils tirent leur alimentation (Umba, 2002), (Zayukua, 2004) et (Umba et Masimango, 2019). Il convient donc de mettre en place des mesures de prévention vis-à-vis de ces divers agents pathogènes appelées mesures de biosécurité qui est en train de devenir l'une des questions les plus urgentes à traiter dans les pays développés, en développement ou en transition (Heskia, 2011). Le concept «une santé » préconise le remaniement des modalités, de gestion des maladies infectieuses alimentaires dans le monde, avec notamment l'institution du principe de précaution et l'évacuation des risques comme fondements des politiques publiques relatives à la sécurité des aliments (FAO, 2007).

ou institutions ressources à travers :

- la revue documentaire,

- $\quad$ les visites de terrain, et enfin

- les consultations et entretiens participatifs auprès des personnes ressources.

3.2 La revue documentaire: La revue documentaire a consisté à collecter des informations de base relatives :

- $\quad$ aux abattages, inspection de la viande et 
maladies zoonotiques ;

- aux caractéristiques biophysiques et humain autour des aires d'abattage ;

- à la législation et réglementation applicables en RD Congo.

3.3 Les visites de terrain : Ces visites du site et des environs avaient entre autres objectifs :

- identifier les établissements, les infrastructures, les équipements et les activités des aires d'abattage ;

- observer les différentes étapes tout au long de la chaîne de transformation de la viande aux aires d'abattage de Matete ;
- $\quad$ analyser les conditions dans lesquelles les animaux sont abattus et vendus dans ces marchés ;

- $\quad$ apprécier la sensibilité environnementale des aires d'abattage.

3.4 Les consultations: Sur la base de guides préétablis, il a été réalisé des entretiens participatifs avec les responsables des services vétérinaires, de l'environnement, les propriétaires des aires d'abattage et les autorités municipales. Ces différents entretiens ont permis de recueillir des informations pertinentes sur les aires d'abattage et les avis et préoccupations des personnalités susmentionnées.

\section{PRESENTATION DE LACOMMUNE DE MATETE}

Matete est l'une des communes de la ville de Kinshasa en République démocratique du Congo qui a pour coordonnées géographiques $4^{\circ} 23^{\prime} 28^{\prime}$ Sud et $15^{\circ} 21^{\prime} 21^{\prime}$ Est dont la superficie est de 488ha, entourée des communes de Lemba, Kinseso, Limete et Ndjili. Elle est séparée de la commune de Ndjili par une rivière qui porte également le nom de sa commune voisine (Ndjili). Elle est composée de trente-six quartiers dont le plus grand est Maindombe qui comporte deux plus grandes écoles publiques. Le quartier Mutoto est le siège administratif de la municipalité (fr.wikipedia.org/wiki / Matete).

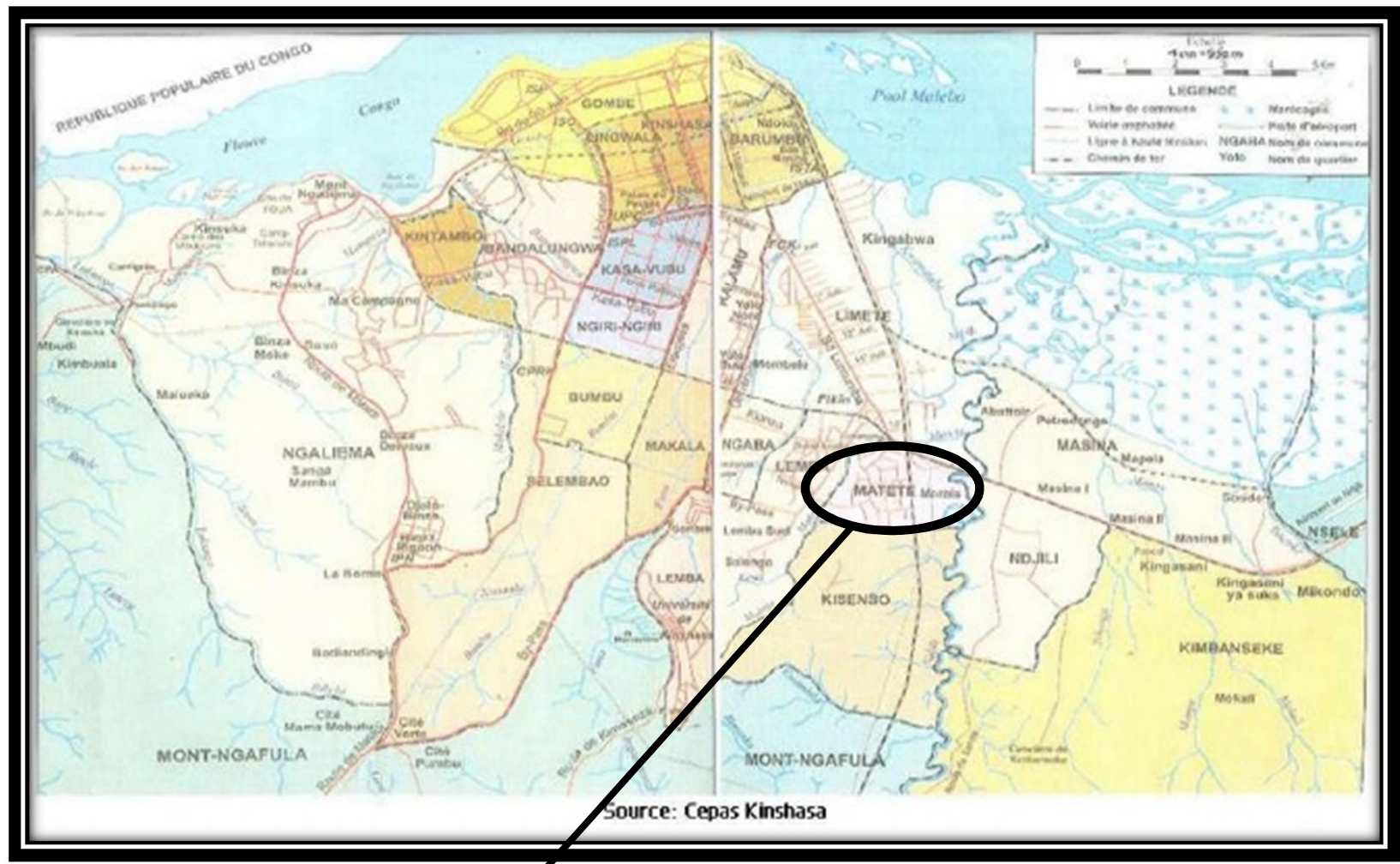

Photo 1 : Localisation de la communde Matete sur la carte administrative de la ville de Kinshasa Source : de Saint Moulin (2011) 


\section{PRESENTATION ET INTERPRETATION DES RESULTATS}

Tenant compte de notre méthodologie de recherche nous avons opté de présenter chaque aire d'abattage.

\subsection{AIRE D'ABATTAGE MPUDI :} L'aire d'abattage de Mpudi $\mathrm{n}^{\circ} 14$ se trouve dans le quartier Mpudi, depuis2010, l'année où la tuerie de la Commune de Matete avait été détruite suite aux travaux d'aménagement du marché central de ladite Commune. Le propriétaire a carrément érigé la tuerie à côté de son domicile prétendant avoir signé un partenariat avec la Commune en offrant son service vétérinaire. Cette aire d'abattage est une petite maison (Photo 2) construite en dur aux dimensions de $5 \mathrm{~m}$ sur $4 \mathrm{~m}$, avec une toiture semiouverte qui se trouve actuellement à un état délabré avec deux pièces dont l'une est destinée comme parc d'attente (stabulation) et l'autre réservée pour le lavage des bêtes grillées. Devant cette maison se trouve un espace libre qui sert de lieu pour griller les bêtes (Photo 3 ). Le système de griller ces bêtes est constitué de 3 jantes de gros véhicule à l'intérieur desquelles on met des morceaux des cartons et des feuilles servant d'allumer le feu; au-dessus de ces jantes on a placé deux barres métalliques et un grillage. La distance qui sépare la tuerie et la maison d'habitation est de $10 \mathrm{~m}$. Notons que cette aire dans la parcelle du propriétaire, n'abat que des porcins.

a) Approvisionnement en eau et en électricité : L'approvisionnement en eau ne pose aucun problème parce qu'il y a une pompe d'eau de la REGIDESO dans la parcelle. Par contre, il n'a pas besoin de l'électricité étant donné que les abattages commencent généralement à 7 h00' et se terminent à $11 \mathrm{~h} 00^{\prime}$ 12h30'.

b) Capacité et fonctionnement hygiénique: Les fournisseurs - abatteurs arrivent très tôt le matin aux environs de 5h30' à 6h00' avec les bêtes. Et les abattages commencent à 7 h30' pour terminer à $11 \mathrm{~h} 00^{\prime}$ au plus tard 12h30' comme dit.

Tableau 1: Nombre de bêtes abattues de janvier - Mars 2019

\begin{tabular}{|l|l|l|l|l|}
\hline Mois & Janvier & Février & Mars & TOTAL \\
\hline Espèces abattues & Porcins & Porcins & Porcins & \\
\hline Nombre de tête & 300 & 224 & 400 & 924 \\
\hline
\end{tabular}

Pour la préparation de ces bêtes, l'aire d'abattage de Mpudi dispose d'un personnel de 10 fournisseurs - abatteurs et 5 bouchers, soit 15 personnes. De façon générale, le personnel est inexpérimenté, n'a pas le niveau de formation requis pour tout manipulateur d'aliments et leur propreté vestimentaire ne tient pas compte des normes et mesures sanitaire. Sauf un seul d'entre eux, en la personne de Baseya qui a travaillé à la ferme DAIPN en 1989, qui a au moins une expérience en matière de boucherie. Au premier trimestre 2019, 924 porcins avaient été abattus .La tuerie a les matériels suivants: couteau, machette, balance, seau, jantes de gros véhicule, barres métalliques, brosse.

c) Evacuation des eaux résiduaires : Il existe un système d'évacuation des eaux usées mais non approprié parce que c'est juste un petit canal qui part de la salle de lavage des bêtes jusque dans le caniveau public qui est souvent bouché. 
Tableau 2: Résume la réalité de l'aire d'abattage MPUDI

\begin{tabular}{|c|c|c|}
\hline Rubriques & Composantes & Constats ou observation \\
\hline $\begin{array}{ll}\text { Milieu } \\
\text { travail }\end{array}$ & $\begin{array}{l}\text {-Abords } \\
\text {-Environnement } \\
\text {-Hygiène de l'aire } \\
\text { d'abattage }\end{array}$ & $\begin{array}{l}\text { - Aire d'abattage est clôturée } \\
\text { - Entourée des forgerons et des vendeurs de viandes et des } \\
\text { divers } \\
\text { - } \quad \text { Assez bonne : présence des mouches et insectes, } \\
\text { pavement et murs moins propres malgré la présence des } \\
\text { étals }\end{array}$ \\
\hline $\begin{array}{l}\text { Matière } \\
\text { première }\end{array}$ & $\begin{array}{l}\text {-Propreté des animaux } \\
\text {-Origine d'animaux } \\
\text {-Etat de santé des } \\
\text { Animaux }\end{array}$ & 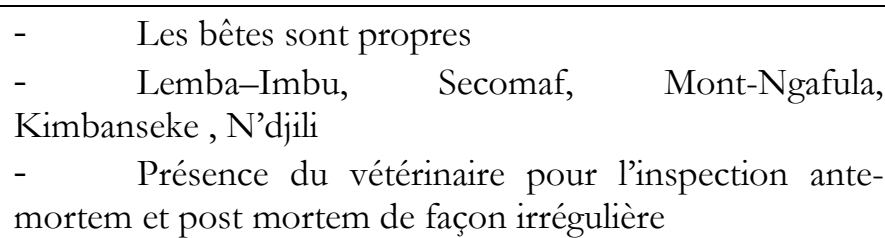 \\
\hline Matériel & $\begin{array}{l}\text {-Matériel de saignée } \\
\text {-Matériel de dépouille } \\
\text {-Entretien hygiénique }\end{array}$ & $\begin{array}{l}\text { - Couteau, machette } \\
\text { - Absence de crochets, présence de jantes de gros } \\
\text { véhicule. } \\
\text { - Les porcs sont dépecés sur les étals en bois } \\
\text { (planches) et l'assommassions se fait sur un sol assez } \\
\text { propre. (Photo 5). }\end{array}$ \\
\hline $\begin{array}{l}\text { Méthode } \\
\text { travail }\end{array}$ & $\begin{array}{ll}- & \text { Stabulation } \\
- & \text { Saignée } \\
- & \\
- & \text { Dépouille et } \\
\text { éviscération } \\
-\quad & \text { Fente de la carcasse } \\
- & \text { Inspection } \\
- & \text { Transport de } \\
\text { viande } & \end{array}$ & $\begin{array}{l}\text { - } \\
\text { en sur nombrésence d'une pièce prévue pour dépôt des bêtes } \\
-\end{array}$ \\
\hline - Main- & $\begin{array}{ll}- & \text { Etat de santé } \\
- & \text { Propreté } \\
\text { vestimentaire } \\
- & \text { Propreté corporelle } \\
- & \text { Sanitaire } \\
- & \text { Formation } \\
\text { professionnelle et } \\
\text { information }\end{array}$ & $\begin{array}{ll}\text { - } & \text { Pas de visite médicale } \\
- & \text { Pas des tenues appropriées mais chacun a sa tenue } \\
- & \text { Normale } \\
\text { - } & \text { Présence de sanitaire dans la parcelle } \\
\text { - } & \text { Non }\end{array}$ \\
\hline
\end{tabular}




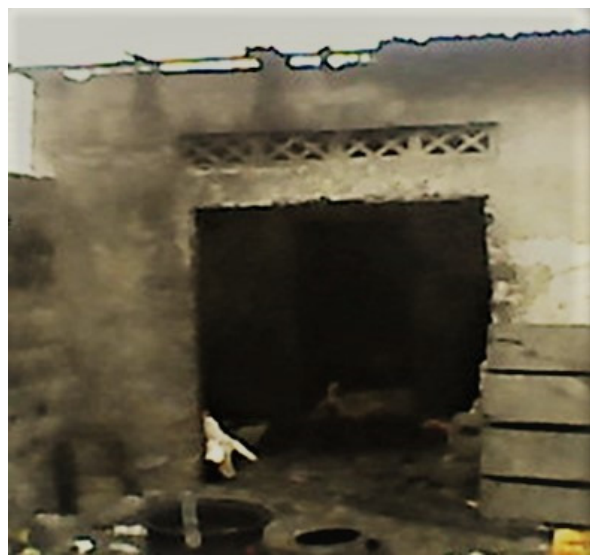

Photo 2 : Aire d'abattage de Mpudi

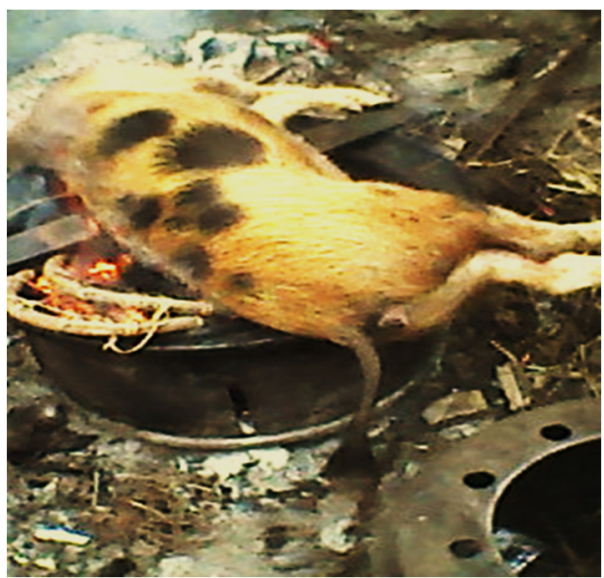

Photo 4:Four pour épiler

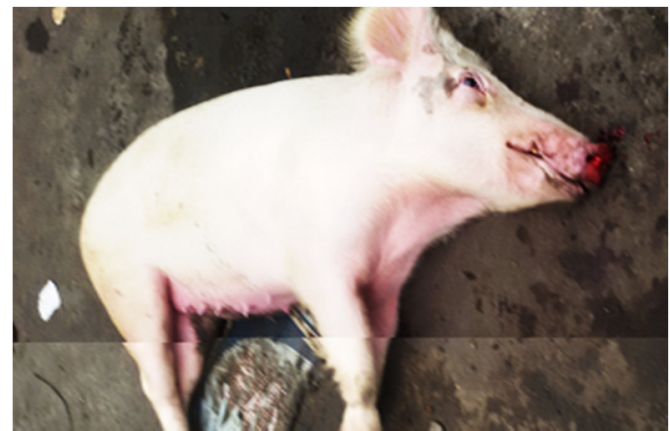

Photo 3 : Porc assommé

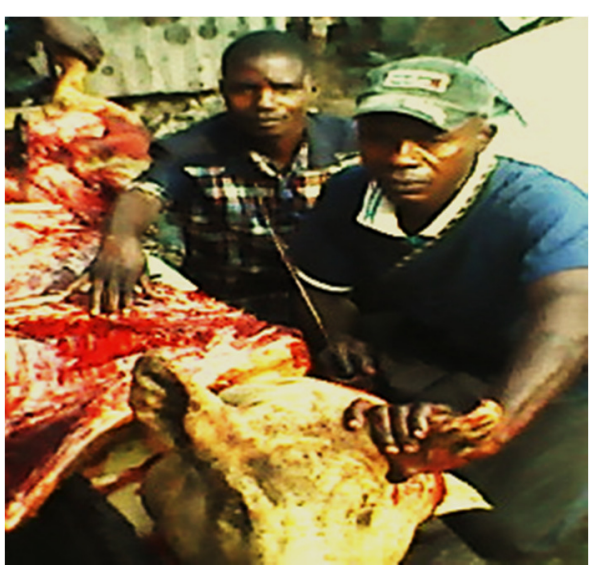

Photo 5 : Porc dépecé

\subsection{AIRE D'ABATTAGE COULOIR} THSILOMBO : L'aire d'abattage couloir Tshilombo est située à l'entrée de l'arrêt Tshilombo, entre les quartiers: LOKORO et NTOMBA. Elle est une initiative privée. Donc, l'aire d'abattage couloir Tshilombo n'abat que les petits ruminants (caprins et ovins) qui proviennent de marché liberté, Mariano, Kongo Central, Kikwit.

a) Approvisionnement en eau et en électricité : L'approvisionnement en eau est bien facile; ils puisent de l'eau dans les parcelles des habitations environnantes. Elle n'a pas d'électricité propre à elle mais elle profite de l'éclairage public du couloir Tshilombo.

b) Capacité et fonctionnement hygiénique : Les fournisseurs leur amènent les bêtes (petits ruminants) au cours de la journée et l'abattage se fait entre $11 \mathrm{~h} 00$ ' et $12 \mathrm{~h} 00$ ', parfois jusqu'à 14h00'. Ils commencent de la viande rôtie (c'est un ordre leur donné par l'autorité municipale). A part les fournisseurs, les abatteurs -rôtisseurs des petits ruminants vont euxmêmes s'approvisionner des bêtes sur pieds au marché de Liberté, à Mariano, aux différents ports de Kinshasa à l'arrivée des embarcations.

Tableau 3 : Nombre des bêtes abattues et rôties de janvier - Mars 2019

\begin{tabular}{|l|l|l|l|l|l|l|c|}
\hline \multicolumn{1}{|c|}{ Mois } & \multicolumn{2}{c|}{ Janvier } & \multicolumn{2}{c|}{ Février } & \multicolumn{2}{c|}{ Mars } & Total \\
\hline Espèce & Ovins & Caprins & Ovins & Caprins & Ovins & Caprins & \\
\hline Nombre de tête & 30 & 150 & 28 & 156 & 19 & 162 & 545 \\
\hline
\end{tabular}

L'aire d'abattage couloir Tshilombo dispose de 4 abatteurs - rôtisseurs et 5 aides, soit 9 personnes, 545 bêtes ont été enregistrées. 
Les abattages se font dans des kiosques (Nganda -Ntaba)

Ce personnel n'a pas de formation professionnelle. Ils ont comme matériels : Couteau, machette, sceau, crochets, cordes, fûts troués, grillages, balance, sceau et bassin (Photo 5)

\section{c) Evacuation des eaux résiduaires}

Il n'existe pas un système d'évacuation proprement dit. Ils utilisent des sachets placés dans des seaux ou bassin pour collecter les eaux usées et les saignées pour enfin les déverser dans des trous préalablement creusés par eux-mêmes.

Tableau 4 : Résume la réalité de l'aire d'abattage couloir Tshilombo

\begin{tabular}{|c|c|c|}
\hline Rubriques & Composantes & Constats ou observation \\
\hline Milieu de travail & $\begin{array}{l}\text { Abords } \\
\text { Environnement } \\
\text { Hygiène de l'aire d'abattage }\end{array}$ & $\begin{array}{l}\text { Aire d'abattage dans des Kiosques non clôturés } \\
\text { Entouré des bars et petits restaurants appelés } \\
\text { "MALEWA" } \\
\text { - assurée : la bête est dépecée et suspendue } \\
\text { à l'aide des crochets ou cordes. }\end{array}$ \\
\hline Matière première & $\begin{array}{l}\text { Propreté des animaux } \\
\text { Origine d'animaux } \\
\text { Etat de santé des animaux }\end{array}$ & 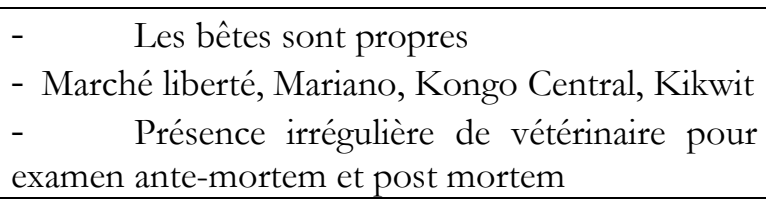 \\
\hline Matériel utilisé & $\begin{array}{ll}- & \text { Matériel de saignée } \\
- & \text { Matériel de dépouille } \\
\text { - } & \text { Entretien hygiénique }\end{array}$ & $\begin{array}{l}\text { - } \quad \text { Couteau, sceau, sachet, machette } \\
\text { - } \quad \text { Crochets, cordes, couteau } \\
\text { - } \quad \text { Normale, car l'abattage se fait en } \\
\text { suspendant la bête coupée en quartiers. Le reste de } \\
\text { quartier suspendu est emballé dans un sachet en } \\
\text { papier bien propre }\end{array}$ \\
\hline Méthode de travail & $\begin{array}{l}- \\
\text { Stabulation } \\
\text { éviscération } \\
\text { Dépouille } \\
\text { Inspection } \\
\text { Fente de la carcasse } \\
\text { - } \quad \text { transport de viande }\end{array}$ & $\begin{array}{l}\text { - N'existe pas parce que la bête est abattue } \\
\text { aussitôt arrivée } \\
-\quad \text { La dépouille existe y compris } \\
\text { l'éviscération, l'animal toujours en suspension. } \\
\text { (Photo 8) } \\
\text { - Ainsi, la fente du sternum et de la carcasse } \\
\text { fait par une machette, la carcasse suspendue } \\
\text { (Photo 9) } \\
\text { - La viande n'est pas transportée mais elle } \\
\text { passe directement à la rôtisserie traditionnelle. }\end{array}$ \\
\hline Main d'œuvre & $\begin{array}{l}\text { - Etat de santé } \\
\text { - Propreté vestimentaire } \\
\text { - Propreté corporelle } \\
\text { - Sanitaire } \\
\text { - Formation professionnelle et } \\
\text { information }\end{array}$ & 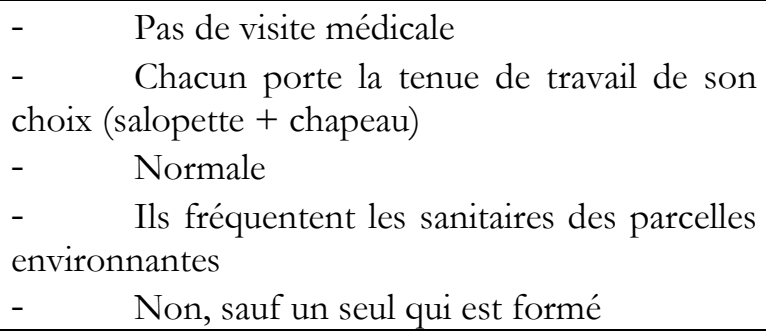 \\
\hline
\end{tabular}




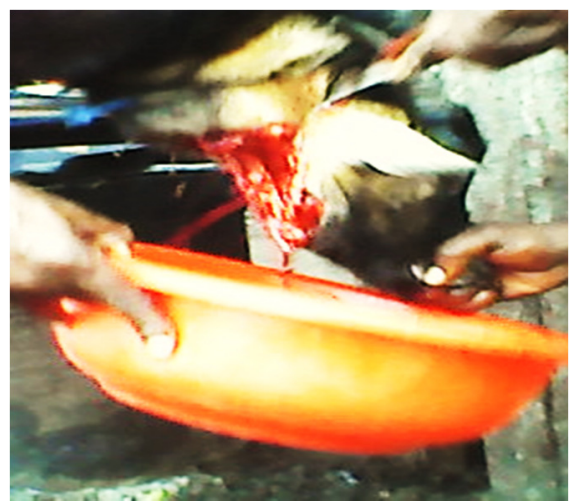

Photo 6: Egorgement dans l'aire d'abattage Couloir Tshilombo

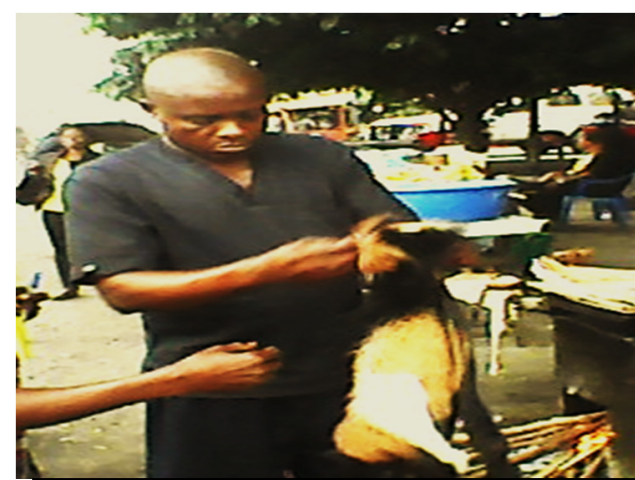

Photo 7: Dépouille dans l'aire d'abattage couloir Tshilombo

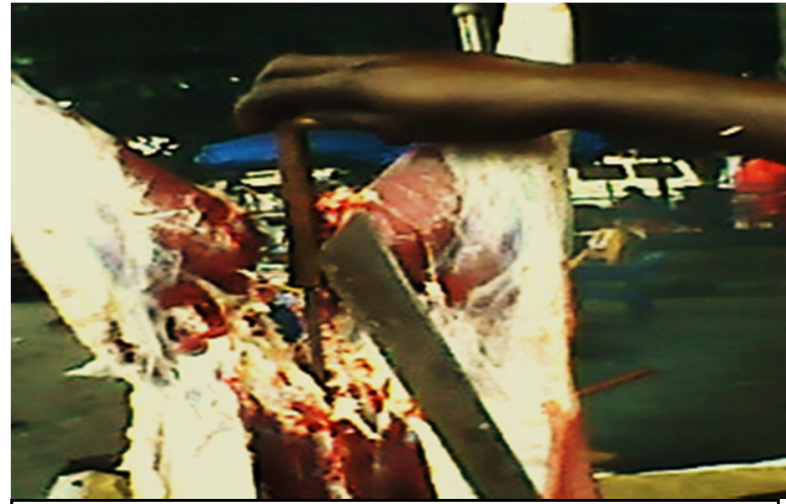

Photo 8: Fente carcasse dans l'aire d'abattage couloir Tshilombo

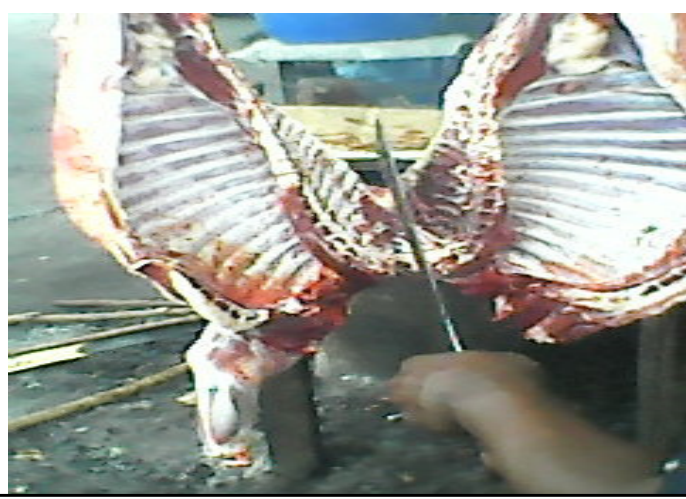

Photo 9 : Fente carcasse du sternum dans l'aire d'abattage couloir Tshilombo
5.3 AIRE D'ABATTAGE NGILIMA : Cette aire d'abattage est située dans le quartier NGILIMA, à côté de l'école primaire Lukeni. Elle est à $5 \mathrm{~m}$ de l'école et à $4 \mathrm{~m}$ de la route séparée par une terrassée. Elle est une initiative privée.

a) Approvisionnement en eaux et en électricité : L'approvisionnement en eau est plus facile grâce à l'eau de la REGIDESO. L'électricité ici n'est pas nécessaire parce que les c)

Tableau 5: Nombre des bêtes abattues de janvier - mars 2019

\begin{tabular}{|c|c|c|c|c|c|c|c|c|c|c|}
\hline Mois & \multicolumn{3}{|c|}{ Janvier } & \multicolumn{3}{c|}{ Février } & \multicolumn{3}{c|}{ Mars } & TOTAL \\
\hline $\begin{array}{c}\text { Espèce } \\
\text { abattue }\end{array}$ & Ovins & Caprin & Porcin & Ovin & Caprin & Porcin & Ovin & Caprin & Porcin & \\
\hline $\begin{array}{c}\text { Nombre } \\
\text { de tête }\end{array}$ & - & - & 30 & - & 5 & 27 & - & 2 & 25 & 89 \\
\hline
\end{tabular}

abattages se font la journée, de 7h00' au plus tard 11 h00' à 12 h00'.

b) Capacité et fonctionnement hygiénique : Les bêtes proviennent soit des fournisseurs soit de leur propre élevage, par ce que Monsieur Donnat est lui-même éleveur des porcs. Cette aire d'abattage de NGILIMA n'abat que l'espèce porcine et rarement les petits ruminants. Ils utilisent comme matériels : machette, couteau, balance, sceau, bassin 
Pour la préparation de ces bêtes, l'aire d'abattage NGILIMA dispose d'un personnel de 3 travailleurs et 1 aide. Ce personnel a un niveau de formation assez bon, 89 bêtes ont été abattues durant cette période. Comme matériels : machette, couteau, balance, sceau, bassin. d) Evacuation des eaux résiduaires : Les eaux usées et les saignées sont collectées dans des sachets placés dans un bassin pour être jetées dans un trou creusé au lieu-même d'abattage.

\section{Tableau 6 : Résume la réalité de l'aire d'abattage NGILIMA}

\begin{tabular}{|c|c|c|}
\hline Rubriques & Composantes & Constat sous observation \\
\hline $\begin{array}{ll}\text { Milieu } & \text { de } \\
\text { travail } & \end{array}$ & $\begin{array}{l}\text { Environnement } \\
\text { Hygiène de l'aire d'abattage }\end{array}$ & $\begin{array}{l}\text { - Aire d'abattage sans maison, sans clôture, } \\
\text { sans kiosques, (en plein air, seulement sous parasol) } \\
\text { (Photos11, 13) } \\
\text { - } \quad \text { Présence de l'école primaire LUKENI } \\
\text { - } \quad \text { Assez bien assurée, car la bête est percée } \\
\text { sur l'étal. }\end{array}$ \\
\hline $\begin{array}{l}\text { Matière } \\
\text { première }\end{array}$ & $\begin{array}{l}\text { Propreté des animaux } \\
\text { Origine d'animaux } \\
\text { Etat de santé des animaux }\end{array}$ & $\begin{array}{l}\text { - } \\
\text { Les bêtes sont propres } \\
\text { Secomaf } \\
\text { - } \quad \text { Le vétérinaire y passe irrégulièrement }\end{array}$ \\
\hline $\begin{array}{l}\text { Matériel } \\
\text { utilisé }\end{array}$ & $\begin{array}{l}\text { Matériel de saignée } \\
\text { Matériel de dépouille } \\
\text { Entretien hygiénique }\end{array}$ & $\begin{array}{l}\text { - Couteau, sceau, bassin, sachets, balance } \\
\text { (Photo 10) } \\
-\quad \text { Cartons et feuilles à bruler à même le sol } \\
-\quad \text { L'abattage se fait sur l'étal propre, couvert } \\
\text { des cartons. (Photo12) }\end{array}$ \\
\hline $\begin{array}{l}\text { Méthode de } \\
\text { travail }\end{array}$ & $\begin{array}{ll}- & \text { Stabulation } \\
- & \text { Dépouillement et } \\
\text { éviscération } \\
- & \text { Inspection } \\
- & \text { Fente de la carcasse } \\
- & \text { Transport viande }\end{array}$ & $\begin{array}{l}\text { - N'existe pas } \\
\text { - Pas de dépouille ; mais l'éviscération se fait } \\
\text { normalement } \\
\text { - Se fait irrégulièrement par le vétérinaire } \\
\text { - Elle se fait à l'aide de la machette } \\
\text { - La viande emballée est transportée dans la } \\
\text { charrette jusqu'au point de vente }\end{array}$ \\
\hline $\begin{array}{l}\text { Main } \\
\text { d'œuvre }\end{array}$ & $\begin{array}{ll}- & \text { Etat de santé } \\
- & \text { Propreté vestimentaire } \\
- & \text { Propreté corporelle } \\
- & \text { Sanitaire } \\
- & \text { Formation professionnelle et } \\
\text { information }\end{array}$ & $\begin{array}{ll}- & \text { Pas de visite médicale } \\
- & \text { Aucune étendue de travail } \\
- & \text { Normale } \\
- & \text { Ils fréquentent les sanitaires d'autrui } \\
- & \text { Aucune formation }\end{array}$ \\
\hline & - & - \\
\hline
\end{tabular}




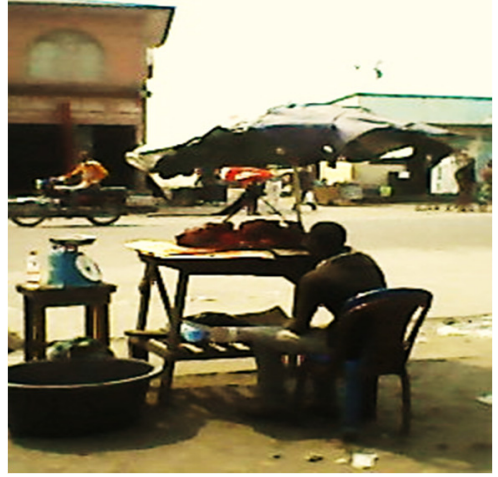

Photo10. Matériels dans l'aire d'abattage de Ndjili

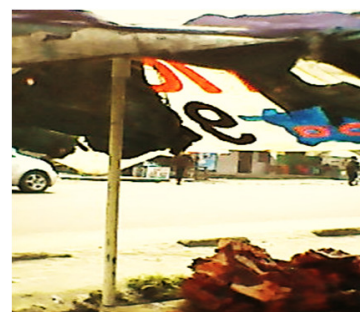

Photo 12: Aire d'abattage Ngilima

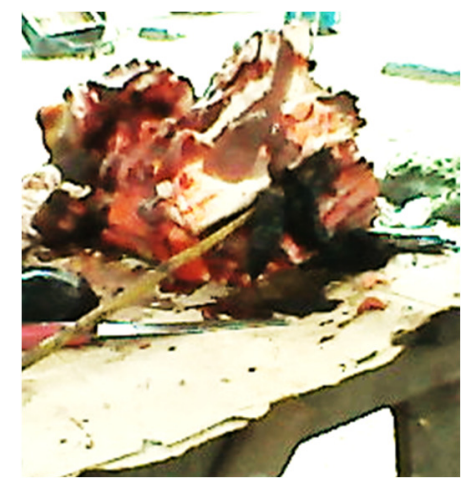

Photo 11. Carcasse sur un étal dans l'aire d'abattage Ndjili

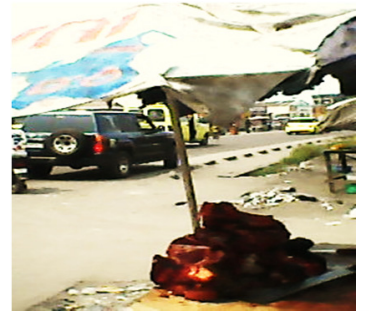

Photo 13 : Viande sous parasol

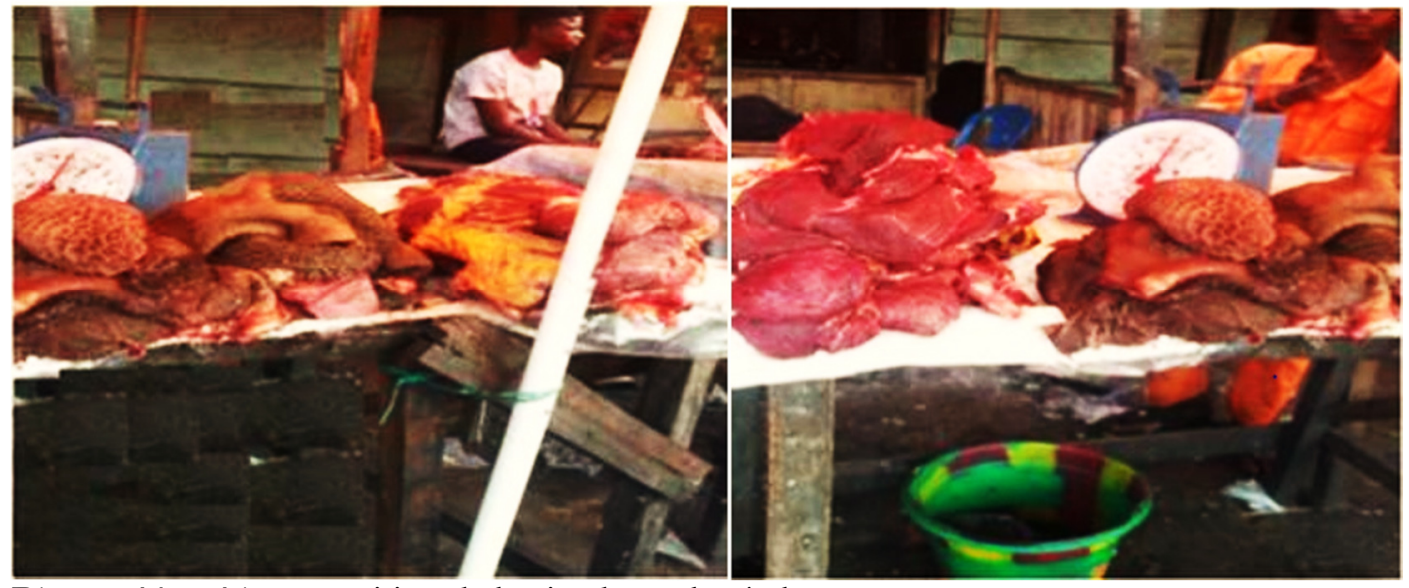

Photos 14 et 15 : Exposition de la viande sur les étalages sans couverture

\subsection{AIRE D'ABATTAGE MAMAN ANNIE}

a) Implantation : L'aire d'abattage Maman Annie est située dans le quartier MPUDI, située le long de la route qui va de KINSAKU vers MALANDI Elle est à une distance de $10 \mathrm{~m}$ des habitations. Le personnel n'est constitué que de la propriétaire et de deux aides, soit 3 personnes. b) Approvisionnement en eau et en électricité : L'approvisionnement en eau ne se pose pas. Car ils utilisent l'eau de la REGIDESO. L'électricité n'est pas nécessaire 
parce que les abattages et la vente se font la journée.

\section{c) Capacité et fonctionnement hygiénique :}

Maman Annie achète ses bêtes (petits ruminants) au marché de Liberté soit les fournisseurs les lui apportent à domicile.

Tableau 7 : Nombre des bêtes abattues de janvier - mars 2019

\begin{tabular}{|l|l|l|l|l|l|l|l|}
\hline Mois & Janvier & Février & Mars & Total \\
\hline Espèce abattue & Ovins & Caprins & Ovins & Caprins & Ovins & Caprins & \\
\hline Nombre de tête & 20 & 10 & 5 & 21 & 3 & 25 & 84 \\
\hline
\end{tabular}

La préparation de ces bêtes est réalisée par la seule propriétaire de cette aire d'abattage et ses deux aides. Tous n'ont pas de niveau de formation requis pour tout manipulateur d'aliments et leur propreté vestimentaire. Le matériel de travail : couteau, machette, sceau et sachets (sachet viva). Un total de 84 bêtes ont été abattues.

d) Evacuation des eaux résiduaires : Il n'y a pas de système d'évacuation des eaux usées comme tel ; on se sert d'un sceau pour collecter les eaux usées et la saignée pour les jeter dans le caniveau.

Tableau 8 : Résume la réalité l'aire d'abattage Maman Annie /MPUDI

\begin{tabular}{|c|c|c|}
\hline Rubriques & Composantes & Constats ou observations \\
\hline $\begin{array}{ll}\text { Milieu } & \text { de } \\
\text { travail } & \end{array}$ & $\begin{array}{l}\text { - Abords } \\
\text { - Environnement } \\
\text { - Hygiène de l'aire } \\
\text { d'abattage }\end{array}$ & $\begin{array}{l}\text { - } \quad \text { Pas de maison, pas de clôture } \\
-\quad \text { Entouré des vendeurs des articles divers et des } \\
\text { "malewa" } \\
\text { - } \quad \text { Bien qu'en plein air sur la route, les alentours sont } \\
\text { un peu propres }\end{array}$ \\
\hline $\begin{array}{l}\text { Matière } \\
\text { première }\end{array}$ & $\begin{array}{l}\text { - Propreté des } \\
\text { animaux } \\
-\quad \text { Origine d'animaux } \\
-\quad \text { Etat de santé des } \\
\text { animaux }\end{array}$ & $\begin{array}{ll}\text { - } & \text { Les animaux sont propres } \\
\text { - } & \text { Marché de Liberté / Masina et les fournisseurs } \\
\text { - } & \text { Présence du vétérinaire mais de façon très irrégulière } \\
\text { pour l'examen ante-mortem et post mortem }\end{array}$ \\
\hline Matériel utilisé & $\begin{array}{l}\text { - Matériel de saignée } \\
-\quad \text { Matériel de } \\
\text { dépouille } \\
-\quad \text { Entretien } \\
\text { hygiénique }\end{array}$ & $\begin{array}{l}\text { Couteau, machette } \\
\text { Couteau } \\
\text { Moins assurée parce que l'abattage se fait à même le }\end{array}$ \\
\hline $\begin{array}{l}\text { Méthode } \\
\text { travail }\end{array}$ & $\begin{array}{l}\text { - } \\
\text { - } \\
\text { éviscération } \\
-\quad \text { Dépouille et } \\
\text { - } \quad \text { Inspection } \\
\text { - } \quad \text { Tente de la carcasse } \\
\text { viande }\end{array}$ & $\begin{array}{l}\text { - } \\
\text { - } \\
\text { N'existe pas parce que l'abattage se fait le même jour. } \\
\text { - } \\
\text { - } \quad \text { Existe mais de manière irrégulière } \\
\text { machette fente de sternum et de la carcasse se fait avec la } \\
\text { - Pas de transport parce que la vente se fait } \\
\text { immédiatement après l'abattage }\end{array}$ \\
\hline $\begin{array}{l}\text { Main } \\
\text { d'œuvre }\end{array}$ & $\begin{array}{l}-\quad \text { Etat de santé } \\
-\quad \quad \quad \text { Propreté } \\
\text { vestimentaire }\end{array}$ & $\begin{array}{l}\text { Pas de visite médicale } \\
\text { Pas de tenue appropriée }\end{array}$ \\
\hline
\end{tabular}




\begin{tabular}{|l|l|ll|}
\hline & $\begin{array}{l}\text { Propreté corporelle } \\
-\end{array}$ & Sanitaire & Normale \\
$-\quad \begin{array}{l}\text { Formation } \\
\text { professionnelle } \\
\text { information }\end{array}$ & et & - & Absence des sanitaires \\
\hline
\end{tabular}

Au cours de la période d'étude, allant de Janvier - Mars 2019,1642 bêtes ont été vendu dans les 4 aires d'abattages suivant cette répartition MPUDI 924, Couloir TSHILOMBO 545, NGILIMA 89 et Maman ANNIE 84. Les aires d'abattage de la commune de Matete sont toutes situées au milieu des agglomérations, ce qui fait l'apparition de bon nombre de nuisances telle que des odeurs des urines et ne sont pas construites dans les normes internationales. La plupart des abattages se font à même le sol, nous constatons que du point de vue évacuation des eaux résiduaires, l'aire d'abattage Tshilombo et Ngilima ont le même système (un trou dans le périmètre de l'aire d'abattage) et Mpudi et Maman Annie déverse dans les caniveaux. L'impact sur l'environnement est particulièrement important puisqu'il s'agit d'établissements nuisant, générant de grandes quantités de rejets liquides (eaux usées, sang) et de rejets solides, sans pour autant qu'ils disposent des moyens adéquats pour gérer ces flux. Nos résultats vont dans le même sens que ceux obtenus par Chabi (2014). La mise à niveau de ces aires d'abattage est indispensable, en majorité se sont des espaces rudimentaires et inadéquats. Cela se justifie par des défauts de leur conception et par la vétusté de leurs infrastructures et équipements. Aussi sur le plan

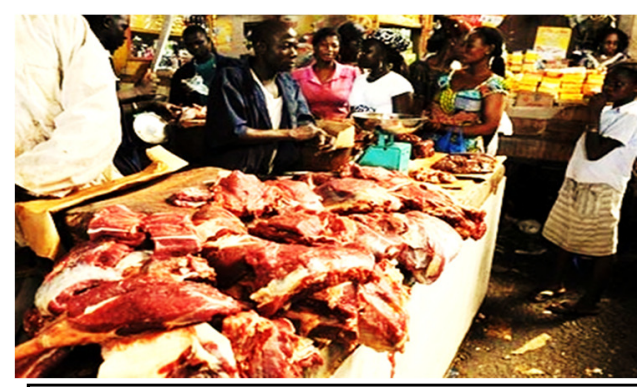

Photo 16 : Viande exposée pendant longtemps sur l'étal sanitaire, une insuffisance de dispositifs de nettoyage et de désinfection est observée. De plus, le manque de personnel qualifié et des irrégularités du contrôle du vétérinaire renvoient à des techniques d'abattage incapables de garantir la qualité hygiénique des viandes, les observations faites durant notre étude rencontrent celles de Hadjé (2014).

Vente et Transport des viandes : Nos enquêtes ont montré que le problème de transport ne se pose pas tellement, car les clients achètent sur le champ quelque temps après l'abattage. Nous pouvons seulement préciser qu'il y a risque de contamination lorsque les mains des abatteurs et / ou des clients sont souillées. Aussi, le transport par moto et charrette qui pourra être la voie de contamination des viandes si les mesures d'hygiène ne sont totalement pas observées car, moto et charrette transportent non seulement les viandes mais aussi les produits non alimentaires .L'exposition à la vente ne se fait pas toujours dans les meilleures conditions d'hygiène, aggravée par le fait que les viandes sont maintenues pendant plusieurs heures à température ambiante favorable aux proliférations microbiennes comme illustré sur la photo13 et photo16. 
Hygiène du personnel : manque manifeste de tenue de travail, L'hygiène vestimentaire : L'hygiène vestimentaire du personnel d'abattage est tout à fait déplorable. Ce personnel est vêtu, le plus souvent, d'habits usagés, recouverts de sang. Certains portent des blouses blanches pendant plusieurs jours sans les nettoyer, cette situation des aires d'abattage de Matete est la même que celle rencontrée au Cameroun, au Congo et au Tchad lors d'une étude menée en 2009 par la FAO (FAO, 2009). L'exploitation ou le responsable des aires d'abattage ne doit pas être «oubli » de nettoyage désinfection des mains du porteur sain après un passage aux toilettes peut engendrer une contamination du produit manipulé par contact avec transmission

\section{CONCLUSION}

Depuis décembre 2019, l'humanité fait face à un mal, une maladie mortelle, la pandémie de Coronavirus 2019 qui a commencé en Chine et atteint la plupart des pays du monde, en particulier ceux de l'Europe et les USA. L'Afrique n'a pas été épargnée, ni la RD Congo, qui est l'un des pays le plus touché du continent avec plus 250 morts. Ce nombre croit de jour en jour. Les responsables politiques et sanitaires ont pris des mesures pour limiter la propagation du virus. Pour qu'un aliment puisse remplir son rôle de couvrir les dépenses énergétiques de l'organisme qui est au travail ou en croissance, il convient qu'il ne soit ni toxique ni malsain c'està-dire qu'il ne contient aucun élément de nature chimique et qu'il soit indemne des germes pathogènes et que son éventuelle teneur en germes banaux soit comprise dans les limites tolérables avec une bonne qualité. La sécurité Sanitaire tout comme la Sécurité Alimentaire devraient constituer une préoccupation majeure non seulement de tous ceux qui assurent un contrôle à différents niveaux de responsabilités en santé publique, mais également des abatteurs ou Consommateur par la suite et ainsi avoir des conséquences dramatiques sur une personne fragile (Mesures Générales d'Hygiène d'HACCP) (Carpier, 2007). Compte tenu des Normes Alimentaires Internationales (Codex Alimenterius), l'OMS et la FAO s'emploient à fournir des avis scientifiques, chimiques et de réduire les risques de transmission des zoonoses à l'homme par la consommation de produits animaux (FAO, 2009). Tout comme des directives régionales pour la Réglementation de la Sécurité Sanitaire des Aliments dans les Etats membres de la SADC, à travers le projet de Sécurité Sanitaire des Aliments - Renforcement des capacités du contrôle.

tout comme des consommateurs qui devraient en faire l'un des critères de choix lors des achats. Dans les pays en voie de développement, obéir à ces règles d'hygiène constitue une véritable gageure. Les difficultés économiques, le climat chaud et humidité favorable à la multiplication bactérienne mais surtout, le faible niveau de conscience et l'éducation sanitaire des populations représentent de redoutables entraves. Il ressort de nos résultats que la présence des différentes aires d'abattage est une conséquence de manque des abattoirs publics. Cette situation entraine un problème de non contrôle de Sécurité Sanitaire de la part de l'Etat et constitue un problème de santé publique. En outre, ces aires d'abattage souffrent de manque de matériel de travail ce qui fait que les viandes livrées sont transportées dans des charrettes utilisées à d'autres fins telles que les immondices et sont vendues dans un environnement insalubre, exposées à l'aire à la merci des mouches, de la poussière et de la fumée provenant des véhicules et motos ou étalées à même le sol. 


\section{RECOMMANDATIONS}

1. Au propriétaire des aires d'abattages

- Mettre leurs aires d'abattage aux conditions idéales de sécurité sanitaire

- A fournir des tenues adéquates aux abatteurs

- Ils doivent toujours travailler en étroite collaboration avec le vétérinaire de façon qu'avant et après l'abattage qu'il soit informé.

\section{Aux abatteurs :}

- $\quad$ Avoir les bonnes pratiques d'hygiène lors de la manipulation de la viande et l'hygiène corporelle, il en eux de même pour les matériels utilisés.

\section{Acheteurs et vendeurs :}

- A bien choisir le site d'achat de la viande

- $\quad$ A dénoncer ou à conseiller les sites qui vendent à côté d'autres produits sans précautions

\section{BIBLIOGRAPHIE}

Bisimwa, P., (2011) Évaluation de l'État sanitaire des vaches abattues à l'abattoir public de Bukavu. Mémoire, Université Évangélique en Afrique, Bukavu, RD Congo, $50 \mathrm{p}$

Borges, F., (2014) Sécurité sanitaire des aliments, Université de Lorraine, $54 \mathrm{p}$

Carpier P, (2007) Mise à jour de la qualité des carcasses et de la viande de bovins adultes, Rapport Final numéro 17 Mai32022 Ministère de la qualité de viande, Département Technique et la qualité de reproduction, pp 12-58.

Chabi Bouko B., (2014) Audit technique des abattoirs de Cotonou/Porto-Novo : Prévalence des motifs de saisies d'abats rouge de bovins et leur incidence socioéconomique Thèse : Méd. Vét. : Dakar ; 08

De Saint Moulin (2011) Atlas de l'organisation administrative de la République Démocratique du Congo, $2^{\text {ème }}$ Édition revue et amplifiée, Centre d'Études Pour l'action Sociale, CEPAS-Kinshasa, $253 \mathrm{p}$

FAO (2009) .Étude sur les abattoirs d'animaux de boucherie en Afrique centrale
- $\quad$ Ils doivent se rassurer de l'origine ou de la provenance de la viande

- La viande achetée doit être rassurée d'une aire d'abattage reconnue officiellement par l'Etat et l'expertise est faite régulièrement par le vétérinaire.

\section{Autorité publique :}

- $\quad$ Que les abatteurs s'organisent en petites associations et l'Etat vient les appuyer

- $\quad$ A encourager les initiatives locales

- A assurer l'inspection effective de ces aires d'abattage.

- De règlementer cette activité nous semble importante vu le nombre de bêtes.

\section{Chercheurs :}

- $\quad$ Faire une étude microbiologique afin de déterminer le niveau de contamination de la viande, des matériels et des infrastructures

$$
\begin{aligned}
& \text { (Cameroun - Congo - Gabon - Tchad) } \\
& 65 \text { pages. }
\end{aligned}
$$

FAO (2013). Étude sur les abattoirs d'animaux de boucherie en Afrique Centrale (Cameroun - Congo - Gabon - Tchad), $70 \mathrm{p}$.

FAO, (2007) : Importance de la biosécurité dans la réduction du risque de grippe dans les élevages et les marchés

Hadje Madina H. (2014): Etat des lieux des abattoirs et aires d'abattage situes dans trois régions du Tchad. Thèse de doctorat en médecine vétérinaire, Université cheikh anta diop de Dakar 161 pages.

Heskia, B., (2011), Hygiène et Biosécurité, in cours de pathologie infectieuse des ruminants, unité de pathologie du bétail. ENVA. Maison- ALFORT

http//www.veternairesaucanada.net/document /une-seule-sante-2006-pdf

https://sante.journaldesfemmes.fr//fichesmaladies/2627507-coronavirus-covid19-groupe-sanguin-o-ab-a-b-risquepourcentage/ 
https://theconversation.com/crise-alimentairecinq-pistes-pour-falsifier-lesapprovisionnemets

https://ww.int/food safety/ares-wak/fr/. https://www.lemonde.fr/planete/article/2020 $\angle 05 / 17 /$ plus-de-cent-cas-de-covid-19detectes-dans-deux-abattoirsfrancaid 603960-3244.html

Morand, S., Figuié, M., (2016) Émergences de maladies infectienses: Risques et enjeux de société. Éditions Quae, RD 10, 78026 Versailles Cedex, France, 133 p.

SADC (2011) Directives Régionales pour la réglementaire de la sécurité sanitaire des aliments dans les états membres de la SADC, Novembre, $46 \mathrm{p}$

Singla, R., Mishra, A., Joshi, R. et al. Human animal interface of SARS-CoV-2 (COVID-19) transmission: a critical appraisal of scientific evidence. $V$ et Res Commun 44, 119-130 (2020). https://doi.org/10.1007/s11259-02009781-0
Umba J. et Masimango T. Analyse de la qualité microbiologique du pain commercialisé à Kinshasa (2019), Editions Universitaires Européennes, is a trademark of international Book Market Service Ltd., member of Omniùscriptum Publiushing Group 17 Meldrum Street, Beau Bassin 71504, Mauritius, $58 \mathrm{p}$

Umba, J., (2002) Contribution à l'étude microbiologique des aliments consommés en l'état à Kinshasa, cas du pain, Mémoire de DES, Faculté des Sciences Agronomiques, Université de Kinshasa, inédit $78 \mathrm{p}$

www.bbc.com/afrique/mone-52974050

www.santelog.com/actualités/Covid-19-de-lachauve

Zayukua BE (2004) Analyse microbiologique des poulets, des Chinchards et des poissons salés vendus à Kinshasa en vue de la sensibilisation à la méthode HACCP, T.F.C, Institut Supérieur AgroVétérinaire de Kimwenza, inédit 50 p 